\title{
Large Numbers of Matings Give Female Field Crickets a Direct Benefit but not a Genetic Benefit
}

\author{
Susan N. Gershman
}

Revised: 24 April 2009 /Accepted: 24 September 2009 /

Published online: 20 October 2009

(C) The Author(s) 2009. This article is published with open access at Springerlink.com

\begin{abstract}
Female crickets can potentially gain both direct and indirect benefits from mating multiple times with different males. Most studies have only examined the effects of small numbers of matings, although female crickets are capable of mating many times. The goal of this paper is to examine the direct and indirect benefits of mating large numbers of times for female reproductive success. In a previous experiment, female Gryllus vocalis were found to gain diminishing direct benefits from mating large numbers of times. In this study I attempt to determine whether mating large numbers of times yields similar diminishing returns on female indirect benefits. Virgin female Gryllus vocalis crickets were assigned to mate five, ten or 15 times with either the same or different males. Females that mated more times gained direct benefits in terms of laying more eggs and more fertilized eggs. Females that mated with different males rather than mating repeatedly with the same male did not have higher offspring hatching success, a result that is contrary to other published results comparing female reproductive success with repeated versus different partners. These results suggest that females that mate large numbers of times fail to gain additional genetic benefits from doing so.
\end{abstract}

Keywords Polyandry $\cdot$ genetic benefits $\cdot$ direct benefits $\cdot$ hatchability $\cdot$ field cricket

S. N. Gershman

Department of Biology, University of California, Riverside, Riverside, CA, USA

S. N. Gershman $(\square)$

School of Biological Sciences, Illinois State University, Normal, IL 61790-4120, USA

e-mail:sgershm@ilstu.edu 


\section{Introduction}

Mating multiple times can be beneficial for females. By mating more than once, females can gain direct benefits (Arnqvist and Nilsson 2000), such as replenishment of depleted sperm stores, access to male-held resources, food and chemical gifts (Vahed 1998). Females can also gain indirect or genetic benefits from mating with multiple partners. Females that have already mated may "trade-up" by mating with or selectively using sperm from successively more genetically beneficial males as they encounter them in the environment. Females may choose mates that provide their mutual offspring with superior genes or females may seek combinations of maternal and paternal genes to avoid genetic incompatibility or increase offspring heterozygosity (Yasui 1998; Jennions and Petrie 2000; Mays and Hill 2004). If male fertilization success is correlated with offspring viability, females that mate multiple times and allow sperm competition to determine offspring paternity will have more viable offspring than females that mate with a single male Sivinski (1984). Alternatively, by genetic "bet-hedging", a female that mates multiple times may potentially improve her fitness by creating genetically diverse offspring that can survive unpredictable environmental conditions in the future (Jennions and Petrie 2000). However, mating with multiple partners can also be costly for females (Sakaluk 1990), thus it is expected that females mate only as often as needed to maximize fitness.

Field cricket females can mate large numbers of times. Based on molecular analysis of wild caught female Gryllus bimaculatus, females may mate as many as seven times (Bretman and Tregenza 2005), and based on an enclosure study, female Gryllodes sigillatus may mate as many times as 15 times in a lifetime (Sakaluk et al. 2002). However, the effects of large numbers of matings on female lifetime reproductive success are generally overlooked (Arnqvist and Nilsson 2000). In past studies of Gryllus vocalis, females that mated many times gained diminishing direct benefits from doing so: females gained fecundity and fertility benefits from mating up to ten times, but failed to gain additional benefits from more matings (Gershman 2007a). However, the consequences of G. vocalis females mating multiply with different partners rather than repeatedly with the same male have not been explored. In this paper, I examine whether females are willing to mate more times than maximizes their direct benefits because these large numbers of matings provide genetic benefits. Alternatively, females may gain diminishing genetic benefits from mating large numbers of times.

One experimental approach used to separate direct from indirect benefits is to control the number of times that females mate, but vary the number of mating partners. This approach holds constant the effect of the direct benefits that females receive from mating, but varies the genetic contributions of partners. If females gain genetic benefits, females mating with multiple partners should have increased offspring viability as compared to females mated with a single partner (Sivinski 1984). Past studies in which female crickets were paired repeatedly with the same partner versus multiply with different partners show that females that mate with more partners have more offspring that successfully hatch (Tregenza and Wedell 1998; Simmons 2001; Fedorka and Mosseau 2002) and/or higher offspring survival to adulthood (Ivy and Sakaluk 2005; Fedorka and Mosseau 2002). However, these 
studies examine only the consequences of mating relatively few times, and the effects of large numbers of matings on genetic benefits are not known.

In this study, to determine whether the indirect benefits of multiple mating decrease as females mate more times, females were assigned to mate five, ten or 15 times either repeatedly with the same male, or multiply with different males. If females receive diminishing genetic benefits from mating with increasing numbers of different males, I predict an effect of partner identity (repeated mate versus different mates) on offspring hatchling success, as well as an interaction between the effect of numbers of matings and partner identity on hatching success.

\section{Methods}

Vocal field crickets, Gryllus vocalis (Weissman et al. 1980), were collected from the Botanic Gardens at the University of California, Riverside. The lab colony was initiated with 50 adult males and 50 adult female crickets in spring 2002, and supplemented each subsequent spring with 30-100 additional field-caught adults. Because of the large numbers of breeding individuals in the colony as well as the frequent supplementation with field-caught individuals (including potentially mated females) the colony has continuously held a high degree of genetic diversity. Crickets were maintained in a growth chamber under simulated summer conditions: $28^{\circ} \mathrm{C}$ with a daily cycle of $14 \mathrm{~h}$ light to $10 \mathrm{~h}$ dark. Animals were given access to moistened cotton for water and oviposition, and maintained on a diet of rabbit chow provided ad libitum.

The basic experimental design was a $3 \times 2$ factorial, with females allowed to mate at one of three levels: five, ten or 15 times, and either mated repeatedly with the same male or multiply with different males. The numbers of matings were chosen based on a previous experiment, in which female Gryllus vocalis were found to mate on average 15 times out of 28 opportunities (Gershman 2007b). The average number of matings was chosen as the "high mating" treatment, because 15 matings is a large number of matings that could reasonably be completed by at least half of the females assigned to this treatment.

Virgin females were randomly assigned to mate five, ten, or 15 times. Females from the five, ten, and 15 mating treatment groups were given 7 days, 14 days and 21 days, respectively, to complete their matings, or they were dropped from the study. This was done to control for the possibility that individual variation in female propensity to mate was associated with other measures of condition such as fecundity or overall health. Thus, females would be equally likely to be dropped from the experiment due to failure to mate their assigned number of matings at all levels of numbers of matings. Females from all treatment groups that died before 21 days were eliminated from the study to prevent females from being differentially eliminated from the treatment groups with more matings.

Three hundred and sixty virgin females were daily given the opportunity to mate with a new male, starting at 5 days after adult eclosion. Mating trials took place at the start of the dark cycle (simulated night) under low light conditions. Females were housed individually in transparent 0.21 cups with constant access to food (rabbit chow) and moist cheesecloth for water and oviposition. For $1 \mathrm{~h}$ a day, females were 
placed with novel males in a clean 0.21 cup. Matings were counted as successful if the copulation resulted in a spermatophore being attached to the female's genital opening. Males were removed from the mating arena after successful copulation to prevent additional matings.

Females in the five, ten and 15 mating treatment groups were assigned to mate with either the same male repeatedly or with novel males for each encounter. In the multiple male treatment groups, males were given a mating opportunity once per night, rotating though the five, ten and 15 mating treatment groups. Males in the multiple mate treatments were used for 3 weeks and then retired. Thus males used with the same partner and males used with different females were on average the same age, and were of similar sexual experience. More females were assigned to the repeated male treatments than the multiple male treatments to compensate for mating pairs dropped from the study due to problems with males, such as male unwillingness to mate or male premature death. Two hundred and fifteen females were assigned to the repeated male treatments, and 145 assigned to mate with multiple males.

Females were provided with moist cheesecloth for oviposition, which was collected every other day until the natural death of the female, and incubated at $28^{\circ} \mathrm{C}$. At $28^{\circ} \mathrm{C}$, Gryllus vocalis eggs generally take $10-15$ days to hatch and embryos that failed to hatch before 15 days died without hatching (personal obs.). After 15 days of incubation, eggs and hatchlings were frozen and counted. If eggs had hatched or contained eyespots or visibly segmented embryos, they were counted as "fertile." Eggs that were either unfertilized or failed to develop to the eyespot stage were counted as "not fertile." It was not possible to visually distinguish unfertilized eggs from fertilized eggs that ceased to develop at the earliest stages, so this measure of fertility is a conservative estimate.

Date of natural death for each female was noted. In analysis of the effects of experimental treatments, only females who had survived at least 21 days (the maximum length of time of the longest treatment group) were included. For the time following completion of experimental treatments until 21 days, females were transferred daily to a clean cup for $1 \mathrm{~h}$ to simulate the handling stress and deprivation of food, water, and oviposition sites experienced by the females that were still receiving mating opportunities. Gryllus vocalis females can have long or short hindwings, and wing length affects fecundity in other orthopteran species (Zera and Denno 1997). Wing morph was recorded for each female. Because larger females can lay more eggs, body size of each female was estimated by measuring pronotum width after death. Although body size and wing morph were included in a preliminary MANCOVA, neither contributed significantly to the model, and were dropped from subsequent analyses.

Fecundity was calculated as the total number of eggs that a female produced in her lifetime. Fertility was calculated as the proportion of fertilized eggs out of the total number of eggs laid per female. Fertility was measured to evaluate whether mating with multiple males provides females with sperm replenishment benefits. Hatchability was calculated as the proportion of hatched eggs per female out of the total number of fertilized eggs per female. Hatchability was included to assess prehatching offspring mortality and determine whether polyandry gives offspring viability benefits. 
Three hundred and sixty virgin females were randomly assigned to the six mating treatment groups (five, ten and 15 matings with the same male; five, ten and 15 matings with different males: $n=70,72,71 ; 49,50,48)$. Ninety females were eliminated because they failed to mate the assigned number of times within the limited period of time allotted $(n=18,19,13 ; 14,13,12)$. Fifty-one females were dropped from the study because although they completed their assigned number of matings, they died before 21 days (the duration of the longest mating trials) was completed $(n=10,9,9 ; 8,7,9)$. Thirty-three females from the repeated mating group were dropped because their assigned mate died before the females had mated their assigned number of times. Eleven females were dropped because they exceeded 3 Mahalanobis distances as multivariate outliers. One hundred and seventy-five females were included in this analysis.

As a proportion, fertility was non-normally distributed and was arcsine square root transformed for normality. Fecundity, fertility, hatchability and survival were standardized to correct for differences in scale among the three dependent variables. The data was screened for outliers using univariate standard deviations and multivariate Mahalanobis distances. Outliers exceeding 1.5 times the interquartile range from the quartiles and outliers exceeding 3 Mahalanobis distances were removed. With these corrections, the data conformed to the assumptions of normality, independence and equality of variance, and lack of multicollinearity among dependent variables.

Multivariate analysis of variance was performed to determine the effect of numbers of matings and mate (same versus different male) on four dependent variables: fecundity (total number of eggs laid), fertility (proportion of fertilized eggs per female out of the total number of eggs laid per female), hatchability (proportion of hatched eggs per female out of the total number of fertilized eggs per female) and survival (female post-experimental survival in days). Multivariate analysis of variance and factorial analysis of variance were performed using SPSS 11, and JMP 5.1.1 software.

\section{Results}

Number of matings had an effect on the MANOVA combined dependent variable of fecundity, fertility, hatchability and survival (Wilks' Lambda $\mathrm{F}_{8,}, 332=3.107 p=$ 0.002). Females that mated more times laid more eggs (Table 1, Fig. 1a). Females that mated more times also had increased fertility (Table 1, Fig. 1b). The number of times that females mated did not have an effect on either the proportion of eggs hatching out of the number of fertilized eggs (Table 1, Fig. 1c), or female postexperimental survival (Table 1, Fig. 1d).

Whether females mated repeatedly with the same partner versus multiply with different partners did not have a combined effect on fecundity, fertility, hatchability and survival (Wilks' Lambda $\mathrm{F}_{4,166}=1.15 p=0.335$ ). On average, females that mated with different partners laid more eggs than females that mated repeatedly with the same partner, but this effect was not statistically significant with correction for multiple comparisons (Table 1, Fig. 1a). The interaction between mate (repeated versus multiple) and number of matings did not have an effect on fecundity, fertility, hatchability and survival (Wilks' Lambda $\mathrm{F}_{8,332}=0.537 p=0.828$ ) (Table 1). 
Table 1 Analysis of Variance for the Effect of Number of Matings (Five, Ten or 15 Matings), Mate (Mating with the Same Male or with Different Males) and the Interaction Between Number of Matings and Mate on Female G. vocalis Fecundity (Number of Eggs Laid), Fertility (Proportion of Laid Eggs that were Fertilized), Hatchability (Proportion of Fertilized Eggs that Hatched) and Post-Experimental Survival

\begin{tabular}{lcccc}
\hline & Fecundity & Fertility & Hatchability & Survival \\
\hline \multicolumn{2}{l}{ Number of matings $(5,10,15)$} & & & \\
$\mathrm{df}$ & 2,182 & 2,181 & 2,181 & 2,185 \\
$\mathrm{~F}$ & 10.52 & 8.32 & 0.407 & 0.0484 \\
$\mathrm{p}$ & $0.0014^{*}$ & $0.0044^{*}$ & 0.524 & 0.826 \\
Mate (same male or different males) & & & \\
$\mathrm{df}$ & 2,182 & 2,181 & 2,181 & 2,185 \\
$\mathrm{~F}$ & 4.11 & 1.14 & 0.072 & 0.135 \\
$\mathrm{p}$ & 0.044 & 0.287 & 0.789 & 0.714 \\
Number of matings & $\times$ mate & & & 3,185 \\
$\mathrm{df}$ & 3,182 & 3,181 & 3,181 & 0.101 \\
$\mathrm{~F}$ & 0.0747 & 0.0122 & 0.025 & 0.751 \\
$\mathrm{p}$ & 0.785 & 0.565 & 0.376 & \\
\hline
\end{tabular}

P-values indicated by asterisks are statistically significant with a sequential Bonferroni correction for multiple comparisons

\section{Discussion}

In this study, female crickets gained direct benefits from mating large numbers of times. Females that mated more times laid more eggs, but only up to a point at which additional matings did not yield additional fecundity benefits. In field crickets, male ejaculate contains not only sperm but also prostaglandin precursors (as well as other substances) that stimulate oviposition in females (Loher and Dambach 1989). These oviposition-stimulating substances could potentially be considered a nuptial gift that delivers a direct benefit for females. However, the results of this, and previous studies (Gershman 2007a) indicate that females appear to be willing to mate more times than is necessary to maximize their reproductive success. Whether this behavior constitutes conflict between the sexes over females' present versus future allocation of reproductive effort or a confluence of interests between males and females in maximizing fecundity begs further examination.

Females that mated more times also laid a higher proportion of fertilized eggs. This effect supports the hypothesis that females may benefit from mating multiple times if their sperm stores become depleted or inviable over time, requiring replacement. The proportion of fertilized eggs that females laid continued to increase with large numbers of matings, suggesting that even large numbers of matings provide additional sperm replenishment benefits for females. This result runs contrary to the traditional expectation that because females could receive a lifetime's supply of sperm in a single mating, additional matings are unnecessary for sperm supply. Further, the saturation point for female benefits of numbers of eggs laid is not found with sperm replenishment. Presumably there exists a point beyond the range of numbers of matings used in this study where the benefits of sperm 
a

a
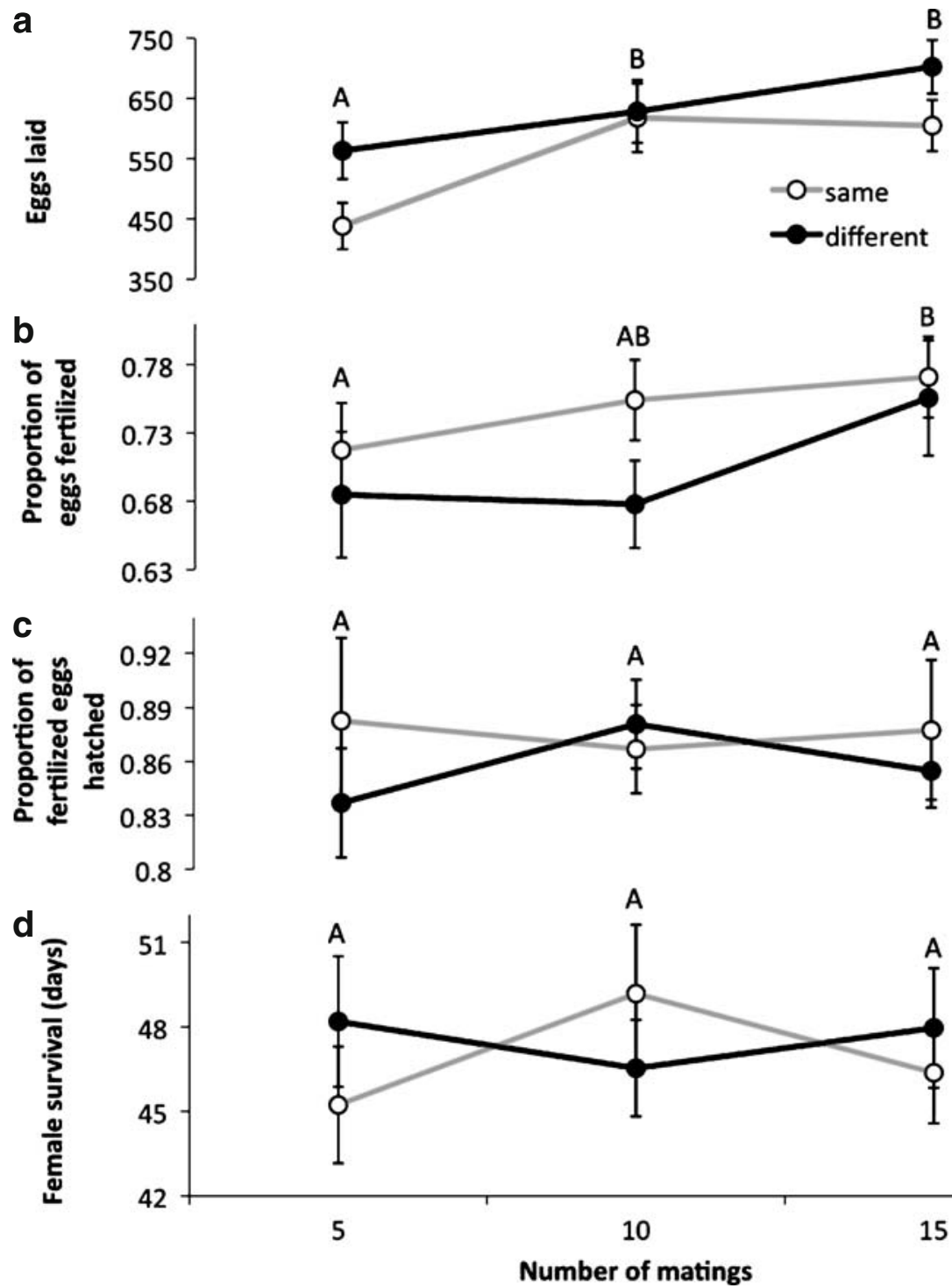

Fig. 1 Means and standard errors for a the total number of eggs laid per females, $\mathbf{b}$ the proportion of eggs laid that were fertilized, $\mathbf{c}$ the proportion of fertilized eggs that hatched, and $\mathbf{d}$ female post-experimental survival. Females mated five, ten or 15 times either repeatedly with the same male repeatedly (open circles) or with different males (dark circles). Comparisons between numbers of matings (pooling females that mated repeatedly with the same male and different males) were conducted using Student-NewmanKeuls tests to control for multiple comparisons. SNK values of $p<0.05$ are indicated by different letters. SNK values of $\mathrm{p} \geq 0.05$ are indicated by the same letter.

replenishment also diminish as female fertility is limited by factors other than sperm depletion or inviability.

Females that mated with different males had a tendency to lay more eggs than females that mated repeatedly with the same male. Although the result was not 
statistically significant in this paper, it has been corroborated by additional research (Gershman 2008a) in which females paired with familiar partners removed male sperm packets faster than females paired with novel partners. If females differentially remove sperm packets when paired with repeated or different partners, selective sperm packet removal likely affects the amount of oviposition-stimulating chemicals transferred from males during copulation, and consequently the numbers of eggs that females lay. If females mating repeatedly with the same partners remove their sperm packets more rapidly, females would likely receive less oviposition-stimulant and consequently lay fewer eggs (Gershman 2008a).

Mating with different males rather than mating repeatedly with the same male did not increase hatchability, the proportion of fertilized eggs that hatch. This result is inconsistent with the results of many published studies in crickets and other taxa in which the offspring of females that mated with multiple partners had a higher viability than the offspring of repeated partners (Simmons 2005; Jennions and Petrie 2000). However, in taxa as diverse as birds, lemon sharks, grasshoppers and field crickets, mating with more than one male failed to increase offspring viability (Caesar and Forsman 2009; Dibattista et al. 2008; Dunn et al. 2009; Jennions et al. 2007; Schmoll et al. 2007). Further, in Drosophila melanogaster, Priest et al. (2008) found that although increasing mating frequency reduced female longevity and fecundity, females that mated at a higher frequency produced daughters with higher lifetime reproductive success (Priest et al. 2008). Thus the effects of polyandry may manifest themselves in later stages of offspring development.

Alternatively, it is possible that mating with more males confers genetic benefits for females, but this effect was not revealed by the experimental design used in this study. If females that have mated with five males have already received the maximum hatchability benefits, they would not gain additional hatchability benefits from mating more than five times. This result rules out a possible motivation for females to mate large numbers of times. In addition, in this study females were provisioned with ad libitum food and water. A previous study on Gryllus vocalis demonstrated that diet can mediate the effect of multiple mating on female fecundity (Gershman 2008b) such that females fed on a low quality diet do not gain fecundity benefits from mating more times. It is possible that diet may also mediate the effect of mating with multiple partners on indirect benefits. If females on a reduced quality diet provision fewer resources to each egg, heterogeneous offspring may be more beneficial than when females are able to provide more provisioning to eggs.

Previous results indicated that females fail to receive additional direct benefits from mating large numbers of times. Further, the results from this paper demonstrate that females also do not gain substantial benefits in hatching success from mating large numbers of times. It is likely that under more hazardous field conditions, environmental influences would set an upper limit to female mating rate. However, although females may be willing to mate more than ten times under laboratory conditions, there are neither substantial costs nor benefits to this behavior.

Acknowledgements I thank Daphne Fairbairn, Marlene Zuk and Derek Roff for their constructive comments. I thank Marlene Zuk and the Zuk lab for advice on experimental design, and Daphne Fairbairn and Derek Roff for their expertise with statistical analysis. Additional material assistance was provided by Daphne Fairbairn and Marlene Zuk. I thank Thuan Vu, Chris Villeneuva, and Veeral Shah for their help as 
lab assistants. Funding for research was provided by the University of California, Riverside Newell and Boyce Fellowships. The experiments performed comply with the law of the United States of America.

Open Access This article is distributed under the terms of the Creative Commons Attribution Noncommercial License which permits any noncommercial use, distribution, and reproduction in any medium, provided the original author(s) and source are credited.

\section{References}

Arnqvist G, Nilsson T (2000) The evolution of polyandry: multiple mating and female fitness in insects. Anim. Behav. 60:145-164

Bretman A, Tregenza T (2005) Measuring polyandry in wild populations: a case study using promiscuous crickets. Molec. Ecol. 14:2169-2179

Caesar S, Forsman A (2009) Do polyandrous pygmy grasshopper females obtain fitness benefits for their offspring? Behav Ecol. 20:354-361

Dibattista JD, Feldheim KA, Gruber SH, Hendry AP (2008) Are indirect genetic benefits associated with polyandry? Testing predictions in a natural population of lemon sharks. Molec. Ecol. 17:783-795

Dunn PO, Lifjeld JT, Whittingham LA (2009) Multiple paternity and offspring quality in tree swallows. Behav. Ecol. Sociobiol. 63:911-922

Fedorka KM, Mosseau TA (2002) Material and genetic benefits of female multiple mating and polyandry. Anim. Behav. 64:361-367

Gershman SN (2007a) Female Gryllus vocalis field crickets gain diminishing returns from increasing numbers of matings. Ethology 113:1099-1106

Gershman SN (2007b) The reproductive consequences of multiple mating and partner novelty in female Gryllus vocalis field crickets. Ph.D. dissertation, University of California, Riverside

Gershman SN (2008a) Post-copulatory female choice increases the fertilization success of novel males in the field cricket, Gryllus vocalis. Evolution 63:67-72

Gershman SN (2008b) Diet quality mediates the effect of multiple mating on female Gryllus vocalis vocal field cricket lifetime reproductive success. Evol. Ecol. Res. 10:269-280

Ivy TM, Sakaluk SK (2005) Polyandry promotes enhanced offspring survival in decorated crickets. Evoution 59:152-159

Jennions MD, Petrie M (2000) Why do females mate multiply? A review of the genetic benefits. Biol. Rev. 75:21-64

Jennions MD, Drayton JM, Brooks R, Hunt J (2007) Do female black field crickets Teleogryllus commodus benefit from polyandry? J. Evol. Biol. 20:1469-1477

Loher W, Dambach M (1989) Reproductive behavior. In: Huber F, Moore TE, Loher W (eds) Cricket behavior and neurobiology. Cornell University Press, Ithaca, pp 43-82

Mays HL, Hill GE (2004) Choosing mates: good genes versus genes that are a good fit. TREE 19:554-559

Priest NK, Galloway LF, Roach DA (2008) Mating frequency and inclusive fitness in Drosophila melanogaster. Amer. Nat. 171:10-21

Sakaluk SK (1990) Sexual selection and predation: Balancing reproductive and survival needs. In: Evans DL, Schmidt JO (eds) Insect defenses: Adaptive mechanisms and strategies of prey and predators. State University of New York Press, Albany, pp 63-90

Sakaluk SK, Schaus JM, Eggert A-K, Sneddon WA, Brady PL (2002) Polyandry and fitness of offspring reared under varying nutritional stress in decorated crickets. Evol. 56:1999-2007

Schmoll T, Schurr FM, Winkel W, Epplen JT, Lubjuhn T (2007) Polyandry in coal tits Parus ater: fitness consequences of putting eggs into multiple genetic baskets. J. Evol. Biol. 20:1115-1125

Simmons LW (2001) Sperm competition and its evolutionary consequences in the insects. Princeton University Press, Princeton

Simmons LW (2005) The evolution of polyandry: sperm competition, sperm selection, and offspring viability. Annu. Rev. Ecol. Evol. Syst. 36:125-146

Sivinski J (1984) Sperm in competition. In: Smith RL (ed) Sperm competition and the evolution of animal mating systems. Academic, London, pp 86-115

Tregenza T, Wedell N (1998) Benefits of multiple mates in the cricket Gryllus bimaculatus. Evol. 52:1726-1730

Vahed K (1998) The function of nuptial feeding in insects: a review of empirical studies. Biol. Rev. 73:4378 
Weissman DB, Rentz DCF, Alexander RD, Loher W (1980) Field cricket (Gryllus and Acheta) of California and Baja California Mexico (Orthoptera:Gryllidae: Gryllinae). Trans. Amer. Ent. Soc. 106:327-355

Yasui Y (1998) The 'genetic benefits' of female multiple mating reconsidered. TREE 12:246-250

Zera AJ, Denno RF (1997) Physiology and ecology of dispersal polymorphism in insects. Annu. Rev. Entomol. 42:207-230 